\title{
Buying and selling price for risky lotteries and expected utility theory with gambling wealth
}

\author{
Michal Lewandowski
}

Published online: 19 July 2014

(C) The Author(s) 2014. This article is published with open access at Springerlink.com

\begin{abstract}
I analyze two expected utility models which abandon the consequentialist assumption of terminal wealth positions. In the expected utility of gambling wealth model, in which initial wealth is allowed to be small, I show that a large WTA/WTP gap is possible and the (Rabin in Econometrica, 68(5), 1281-1292, 2000) paradox may be resolved. Within the same model the classical preference reversal which allows arbitrage is not possible, whereas preference reversal (involving buying prices in place of selling prices), which does not allow arbitrage, is possible. In the expected utility of wealth changes model, in which there is no initial wealth, I show that both a WTA/WTP gap as well as the classical preference reversal are possible due to loss aversion, both in its general as well as some specific forms.
\end{abstract}

Keywords Expected utility · Narrow framing · Rabin (2000) paradox · Preference reversal · WTA/WTP disparity · Buying and selling price for a lottery

JEL Classifications D81 · D03 · C91

Willingness to accept (WTA) or selling price for a lottery is a minimal sure amount of money which a person is willing to accept to forego the right to play the lottery. Willingness to pay (WTP) or buying price for a lottery, on the other hand, is a maximal sure amount of money which a person is willing to pay in order to get the right to play the lottery. The disparity between willingness to pay and willingness to accept is a well-known phenomenon that arises in experimental settings. There is a large body of evidence starting with Knetsch and Sinden (1984) and Thaler (1980) that WTA is much higher than WTP for many types of goods. Horowitz and McConnell (2002)

M. Lewandowski ( $\square)$

Warsaw School of Economics, Warsaw, Masovian, Poland

e-mail: michal.lewandowski@sgh.waw.pl 
is a survey which documents and analyzes results from a great number of experiments and obtains mean values of the WTA/WTP ratio for different goods. The mean WTA/WTP ratio for lotteries is 2.10 and it is small as compared to the same ratio for other goods, especially non-market goods for which the average WTA/WTP ratio is over 7. On the other hand Chilton et al. (2012) find that in the area of medical services as the severity of health complaint is reduced, the WTA/WTP ratio converges across the sample and does not exceed 1 significantly.

Despite the differences across different contexts and goods, WTA is on average much higher in value than WTP. There have been many attempts to give account for this phenomenon.

There is strong belief in the literature that this evidence is not consistent with expected utility theory. Along the lines of Rubinstein (2006) I will argue that the source of this belief lies in associating expected utility theory with the doctrine of consequentialism, according to which "the decision maker makes all decisions having in mind a preference relation over the same set of final consequences". This association is harmless when considering Constant Absolute Risk Aversion (CARA), as in this case decisions whether to accept a given lottery do not depend on wealth. However, as many studies confirm, people usually exhibit Decreasing Absolute Risk Aversion (DARA), in which case wealth effects are present.

In practice the doctrine of consequentialism means that the initial wealth underlying any decision whether to accept or reject a given lottery is assumed to be the decision maker's lifetime wealth. It follows that most lotteries under consideration are small relative to initial wealth and therefore, by Rabin (2000)'s argument for any reasonable level of risk aversion expected utility predicts approximate risk neutrality towards such lotteries. In this case, not only is expected utility incapable of accommodating large spreads between buying and selling price, but also it is inconsistent with risk averse behavior for small gambles ${ }^{1}$. Instead of burying expected utility theory I propose to divorce it from the doctrine of consequentialism, i.e. relax the assumption that initial wealth underlying any decision whether to accept a gamble is total lifetime wealth of the decision maker. If initial wealth is allowed to be small, I will show that expected utility is consistent with large buying/selling price spreads, i.e. that within expected utility for levels of risk aversion consistent with experimental evidence, one can obtain a buying/selling price spread of the magnitude observed in experiments. Following this finding I will propose an alternative for consequentialism involving narrow framing. Instead of asserting that preferences are always defined over total lifetime wealth, I will assume that preferences over gambling are defined over gambling wealth, i.e this part of the decision maker's total wealth which he designates for taking gambles. The idea is taken from Foster and Hart (2009), although the seeds of this approach, and in particular the idea of separating lifetime wealth and something else for different decision problems, are already in Rubinstein (2006).

There are many papers on the disparity between willingness to accept and willingness to pay for risky lotteries. It is part of a vast literature stream on WTA and WTP valuations in general. For example, Schmidt et al. (2008) explain the WTA/WTP

\footnotetext{
1 "Small" here means "small relative to lifetime wealth".
} 
spread for risky lotteries using prospect theory. They propose the third-generation prospect theory, in which, unlike in the previous versions, the reference point is allowed to be random. They show that loss aversion in such a model implies a positive WTA/WTP gap. In a recent paper Viscusi and Huber (2012) show on the basis of a large experiment that in the case of environmental health risk probabilities the WTA/WTP gap is due largely to the reference dependence effects related to costs. Estimated income effects in their results were negligible.

In general, there have been many accounts for the disparity based on non-expected utility models. My aim in this paper is not to offer a better explanation. My goal is to show that large spreads between WTA and WTP, due entirely to wealth effects, are possible within expected utility if only wealth is interpreted narrowly as gambling wealth. The advantage of this approach is to show that one does not have to depart from rationality so much (as in many behavioral models) in order to explain WTA/WTP gaps as well as some other behavioral phenomena.

The approach I take in this paper in general is not novel. As I mentioned before, Rubinstein (2006) claims that a lot of recent confusion around expected utility, which led some researchers to question it as a descriptive theory, is caused by associating expected utility theory with the assumption of consequentialism-the idea that there is a single preference relation over the set of all possible lotteries with prizes being "final wealth levels". For more discussion on the related literature please refer to Section 2.3 on Rabin's paradox. My approach is to apply this reasoning which emerged when discussing Rabin's paradox to a wider spectrum of topics, such as for example the WTA/WTP gap. For more discussion on the doctrine of consequentialism and different alternatives to it, see Section 4.

Related to buying/selling price disparity is the issue of preference reversal analyzed by Grether and Plott (1979). There are two lotteries called the \$-bet and the P-bet both of which promise some prize with some probability and nothing otherwise, such that the probability of winning is higher for the P-bet but the prize is bigger for the \$-bet. Preference reversal occurs when selling price for the \$-bet is higher than that for the P-bet but the P-bet is preferred to the $\$$-bet in a direct choice ${ }^{2}$. A related possibility, which I call preference reversal $\mathrm{B}$, occurs when the buying price for the P-bet is higher than that for the \$-bet and yet the \$-bet is chosen over the P-bet in a direct choice. I will show that traditional preference reversal is susceptible to arbitrage and is not possible within expected utility, whereas preference reversal $\mathrm{B}$ is possible within expected utility and it does not allow arbitrage. This result may suggest that traditional preference reversal is less rational than preference reversal B.

The structure of this paper is as follows. In Section 1 I introduce the model, its assumptions, definitions of buying and selling price for a lottery and buying/selling price reversal. Then I state a couple of technical propositions which describe the

\footnotetext{
${ }^{2}$ Experimentally, in order to confirm preference reversal one must show that the asymmetry described above occurs more often than the opposite kind of asymmetry, i.e. when the $\$$-bet is preferred in a direct choice but the P-bet gets a higher selling price.
} 
shape and properties of the buying and selling price for a lottery for different risk attitudes. Section 2 contains the main theses of the paper. Focusing on the constant relative risk aversion (CRRA) class of utility functions, I demonstrate first that expected utility with consequentialism is likely to predict risk neutral behavior towards most gambles and eventually a gap between buying and selling price becomes negligible. Second, I demonstrate that if the doctrine of consequentialism is abandoned and wealth is allowed to move over the whole domain, significant spreads between buying and selling price are possible due to income effects when wealth is sufficiently small. As a next step, I propose an alternative to consequentialism involving narrow framing. Instead of defining wealth as total lifetime wealth of the decision maker I suggest using gambling wealth, which is that part of the decision maker's total wealth that he designates for the purpose of taking gambles. I discuss ways to test this gambling wealth hypothesis. Then I compare gambling wealth to the concept of pocket cash money introduced by Fudenberg and Levine (2006) and show the similarities and differences between the two ideas. I also mention ways of resolving Rabin's paradox in the literature and how it relates to the concept of gambling wealth and the concept of pocket cash money. In Section 3 I examine the possibility of what I call preference reversal B, which I compare to the related concept of traditional preference reversal. I show that whereas preference reversal B is possible within an expected utility framework with gambling wealth instead of total lifetime wealth and it does not allow arbitrage opportunities, preference reversal allows arbitrage opportunities and is not possible within expected utility. Section 4 contains the discussion on consequentialism, the concept around which much of the discussion and arguments in this paper are organized. This section also discusses different alternatives to consequentialism. Section 5 discusses expected utility over wealth changes as one of these alternatives departing from consequentialism more radically than expected utility with gambling wealth. The issues of WTA/WTP disparity and preference reversal are discussed for this model and the results are compared with similar results for expected utility with gambling wealth. Section 6 concludes. The Appendix at the end of this paper contains proofs of the propositions.

\section{The model}

\subsection{Basic definitions and assumptions}

I start with basic assumptions and definitions.

Assumption 1 Preferences obey expected utility axioms. Bernoulli utility function $U: \mathbb{R} \rightarrow \mathbb{R}$ is twice continuously differentiable, strictly increasing and strictly concave.

Definition 1 A lottery $\mathbf{x}$ is a real- and finite-valued random variable with finite support. The space of all lotteries will be denoted $\mathcal{X}$. I define the maximal loss of lottery $\mathbf{x}$ as: $\min (\mathbf{x})=\min \operatorname{supp}(\mathbf{x})$. 
The typical lottery will be denoted as $\mathbf{x} \equiv\left(x_{1}, p_{1} ; \ldots ; x_{n}, p_{n}\right)$, where $x_{i} \in \mathbb{R}, i \in$ $\{1,2, \ldots, n\}$ are outcomes and $p_{i} \in[0,1], i \in\{1,2, \ldots, n\}$ the corresponding probabilities. Outcomes should be interpreted here as monetary values. Although most results that follow are true for more general lotteries, the finite support assumption is sufficient for the purposes of this paper. Now I define buying and selling price for a lottery given wealth level along the lines of Raiffa (1968). To avoid repetitions, I will henceforth skip statements of the form: "Given utility function $U$ satisfying Assumption 1, any lottery $\mathbf{x}$ and wealth $W . . . "$.

Definition 2 I define selling price and buying price for a lottery $\mathbf{x}$ at wealth $W$ as functions of wealth $W$ denoted, respectively, $S(W, \mathbf{x})$ and $B(W, \mathbf{x})$. Provided that they exist, values of these functions will be determined by the following equations:

$$
\begin{aligned}
& \mathrm{E} U[W+\mathbf{x}]=U[W+S(W, \mathbf{x})] \\
& \mathrm{E} U[W+\mathbf{x}-B(W, \mathbf{x})]=U(W)
\end{aligned}
$$

If utility function is defined over the whole real line as is the case for constant absolute risk aversion, buying and selling price as functions of wealth exists for any wealth level by Assumption 1. If the domain of the utility function is restricted to a part of the real line as is the case of the constant relative risk aversion utility function analyzed here, I will specify later on in the paper on which domain buying and selling price are defined as functions of wealth.

In economic terms, given an individual with initial wealth $W$ whose preferences are represented by utility function $U(\cdot), S(W, \mathbf{x})$ is the minimal amount of money which he demands for giving up lottery $\mathbf{x}$. Similarly, $B(W, \mathbf{x})$ is the maximal amount of money which he is willing to pay in order to play lottery $\mathbf{x}$. Additionally I define a concept of buying/selling price reversal.

Definition 3 Given two lotteries $\mathbf{x}$ and $\mathbf{y}$ and some wealth level $W$, define buying/selling price reversal as:

$$
S(W, \mathbf{y})>S(W, \mathbf{x}) \text { and } B(W, \mathbf{x})>B(W, \mathbf{y})
$$

This kind of preference pattern may be interpreted as follows. For a given initial wealth, an individual's certainty equivalent for lottery $\mathbf{y}$ is higher than for lottery $\mathbf{x}$, and yet he is willing to pay more to play lottery $\mathbf{x}$ than to play lottery $\mathbf{y}$. In other words, an individual exhibiting buying/selling price reversal may prefer to buy $\mathbf{x}$ than $\mathbf{y}$ if he does not have the right to play any lottery initially. When, on the other hand, he does have the right to play the lottery initially, he would prefer to sell $\mathbf{x}$ than $\mathbf{y}$.

\subsection{Preliminary results}

Before introducing the main point of this paper I need a couple of theoretical results which describe properties of buying and selling price for a lottery for different risk 
attitudes. The most basic property of buying and selling price which is true for any concave strictly increasing utility function is the following:

Proposition 1.1 (Concave) For any non-degenerate lottery $\mathbf{x}$ and any wealth $W$ such that buying and selling price exist, $S(W, \mathbf{x})$ and $B(W, \mathbf{x})$ lie in the interval $(\min (\mathbf{x}), \mathbf{E}(\mathbf{x}))$. For a degenerate lottery $\mathbf{x}, S(W, \mathbf{x})=B(W, \mathbf{x})=x$.

Proof In the Appendix.

Below I state propositions which characterize CARA, DARA and CRRA utility functions in terms of buying and selling price. Proofs of these propositions may be found for example in Lewandowski (2011) along with an extensive discussion on multiplicative and nominal gambles and risk aversion notions for the two kinds of gambles. I start with the results on CARA and DARA utility functions:

Proposition 1.2 (CARA) The following two statements are equivalent:

i. Bernoulli utility function exhibits CARA

ii. Buying and selling price are independent from wealth and equal i.e.

$$
B(W, \mathbf{x})=S(W, \mathbf{x})=C_{\alpha}, \forall W
$$

where $\alpha$ is absolute risk aversion coefficient and $C_{\alpha}$ takes real values and depends only on $\alpha$.

Proposition 1.3 (DARA) The following two statements are equivalent:

i. Bernoulli utility function exhibits DARA

ii. $\quad$ buying and selling price are increasing in $W$

$$
B(W, \mathbf{x})>0 \Longleftrightarrow B(W, \mathbf{x})<S(W, \mathbf{x})
$$

for a non-degenerate lottery $\mathbf{x}$.

The above propositions show that in the expected utility model a gap between buying and selling price can only arise due to wealth effects. Selling price is higher than buying price for a lottery for which I would be willing to pay a positive amount only if absolute risk aversion decreases in wealth. In the subsequent analysis I will be especially interested in the CRRA class, which is a subset of DARA:

Proposition 1.4 (CRRA) The following two statements are equivalent:

i. Bernoulli utility function exhibits CRRA

ii. buying and selling price for any lottery are homogeneous of degree one i.e.

$$
\begin{aligned}
& S(\lambda W, \lambda \mathbf{x})=\lambda S(W, \mathbf{x}), \forall \lambda>0 \\
& B(\lambda W, \lambda \mathbf{x})=\lambda B(W, \mathbf{x}), \forall \lambda>0
\end{aligned}
$$




\section{Buying/selling price spread within the expected utility framework}

\subsection{Buying and selling price for CRRA class of utility functions}

In this section I focus on the constant relative risk aversion utility class, since it is simple and empirically well validated. For convenience but without loss of generality I normalize the Bernoulli utility function as follows:

$$
U_{\alpha}(x)=\left\{\begin{array}{lrl}
\frac{x^{1-\alpha}-1}{1-\alpha}, & 1 \neq \alpha>0, & x>0 \\
\log x, & \alpha & =1, x>0
\end{array}\right.
$$

Parameter $\alpha$ is required to be bounded. I also focus on non-degenerate lotteries with non-negative values such that outcome zero gets positive probability. This restriction is a matter of convenience as the forthcoming results extend to the case of general lotteries. The following proposition is necessary to establish the domain and the range of buying and selling price for a lottery as functions of wealth for the case of CRRA functions of the above form. Before I state this proposition a couple of remarks might be useful. First, since the CRRA utility function used in this section is defined only for positive real numbers I need to be sure that both sides of Eqs. 2 and 1 defining buying and selling price are well defined. Second, notice that the CRRA function of the above form is unbounded from below for $\alpha \geq 1$ and bounded from below for $0<\alpha<1$. This is the reason why for $0<\alpha<1$ the infimum of $B(W, \mathbf{x})$ and $S(W, \mathbf{x})$ cannot be equal to $\min (\mathbf{x})$, the lower bound given in Proposition 1.1. It turns out that there is a certain threshold denoted by $W_{L}(\mathbf{x}) \in(0, \mathrm{E}[\mathbf{x}])$ such that the infimum of $B(W, \mathbf{x})$ and $S(W, \mathbf{x})$ is equal to $W_{L}(\mathbf{x})+\min (\mathbf{x})$ which is greater than $\min (\mathbf{x})$.

Proposition 2.1 (CRRA2) Given the class of CRRA utility functions of the form given by Eq. 3 the following holds for any non-degenerate lottery $\mathbf{x}$ : for $\alpha \geq 1$

- $\lim _{W \rightarrow 0} B(W, \mathbf{x})=\min (\mathbf{x})$

- $\lim _{W \rightarrow-\min (\mathbf{x})} S(W, \mathbf{x})=\min (\mathbf{x})$

Define $W_{L}(\mathbf{x})=U^{-1}[\mathrm{E} U(-\min (\mathbf{x})+\mathbf{x})]$. For $0<\alpha<1$

- $\lim _{W \rightarrow W_{L}(\mathbf{x})} B(W, \mathbf{x})=W_{L}(\mathbf{x})+\min (\mathbf{x})$,

- $\lim _{W \rightarrow-\min (\mathbf{x})} S(W, \mathbf{x})=W_{L}(\mathbf{x})+\min (\mathbf{x})$

Additionally,

$$
\forall \alpha>0 \lim _{W \rightarrow \infty} B(W, \mathbf{x})=\lim _{W \rightarrow \infty} S(W, \mathbf{x})=\mathrm{E}[\mathbf{x}]
$$

Proof In the Appendix.

The above proposition establishes the domain and the range of buying and selling price for a given lottery $\mathbf{x}$ as functions of wealth for CRRA utility functions which are defined above. Now that I have introduced the necessary theoretical results, I proceed to the main message of this paper. 


\subsection{Expected utility and consequentialism}

Consequentialism is a doctrine that says that an individual makes all decisions according to a preference relation defined over one set of final consequences ${ }^{3}$. In practice it means that initial wealth taken into account when making whatever decision is interpreted as the decision maker's total lifetime wealth. Most lotteries which a person may encounter are small relative to his lifetime wealth. In particular, lotteries used in experiments have values which are small relative to total lifetime wealth of experimental subjects. Therefore, to explain certain experimental results, it is sufficient to focus on lotteries that have values which are negligible as compared to total lifetime wealth. To represent this fact I assert here that lotteries have bounded values and consequentialism approximately means that wealth tends to infinity. In this case the following result holds:

Proposition 2.2 Expected utility with consequentialism and CRRA approximately predicts no buying/selling price spread and risk neutrality.

Proof The proof follows directly from Eq. 4 in Proposition 2.1. We claim that consequentialism implies that most lotteries encountered in practical decision-making of a given individual have values which are very small compared to his/her lifetime wealth. We shall represent this fact approximately by allowing wealth to go to infinity with lottery values being bounded and fixed. What happens is that both selling price and buying price tend to $\mathrm{E}[\mathbf{x}]$ and hence the gap between them vanishes. Since the distance $\mathrm{E}[\mathbf{x}]-S(W, \mathbf{x})$ measures risk aversion, it is clear that there is no risk aversion either.

This proposition is very similar to Rabin (2000)'s calibration theorem confined to the CRRA class of utility functions. Reasonable levels of risk aversion for big gambles give rise to risk neutral behavior towards small gambles within expected utility with consequentialism. The difference between (Rabin 2000)'s argument is that I claim after Rubinstein (2006) that this is due to consequentialism and not due to expected utility itself.

This negative result immediately raises the issue of what happens if I drop the assumption of consequentialism. To answer this question I proceed in two steps. First, I show that relaxing consequentialism is promising, i.e. a large buying/selling price for a lottery for reasonable levels of risk aversion may be obtained. Second, I propose an alternative assumption which could replace the assumption of consequentialism.

In the first step I allow wealth to vary freely. I will therefore analyze buying and selling price for a lottery as functions of wealth. The goal is to see for what values of wealth is the spread between buying and selling price likely to be high.

\footnotetext{
${ }^{3}$ For an extensive discussion on consequentialism, its precise meaning and different alternatives to it, refer to Section 4.
} 
To save on notation, given a fixed lottery $\mathbf{x}$ I shall write $S(W, \mathbf{x})=S(W)$ and $B(W, \mathbf{x})=B(W)$. I define relative spread between buying and selling price as follows:

$$
\tau(W)=\frac{S(W)-B(W)}{B(W)}
$$

The following lemma can be used to infer certain properties of the relative gap between buying and selling price.

Lemma 1 For a differentiable decreasing absolute risk aversion utility function, given any non-degenerate lottery $\mathbf{x}$ and any wealth level $W$, the following holds:

- $B^{\prime}(W)<1$

- $S^{\prime}(W-B(W))=\frac{B^{\prime}(W)}{1-B^{\prime}(W)}$ and hence $S^{\prime}(W-B(W))>B^{\prime}(W)$

- $B^{\prime}(W+S(W))=\frac{S^{\prime}(W)}{1+S^{\prime}(W)}$ and hence $B^{\prime}(W+S(W))<S^{\prime}(W)$

- $S^{\prime}(W)=\frac{S(W)-B(W)}{B(W)}$ and $B^{\prime}(W)=\frac{S(W)-B(W)}{S(W)}$ for small positive $S(W)$

Proof In the Appendix.

Observe that the slope of buying price is always smaller than one whereas the slope of selling price can be higher for small values of wealth. Before I state a proposition describing the characteristics of the relative gap between buying and selling price I need the following lemma:

Lemma 2 For a CRRA utility function, given any non-degenerate lottery $\mathbf{x}, S(W)$ and $B(W)$ are concave functions.

Proof See Lewandowski (2011).

I focus now on the case when $S(W)>B(W)>0$. The remaining cases can be analyzed similarly. By Proposition 2.1, to make sure that $B(W)$ is positive I require that $\min (\mathbf{x})$ cannot be lower than zero. The following proposition suggests that for a CRRA utility function, the lower the wealth the higher the relative gap between buying and selling price.

Proposition 2.3 For a CRRA utility function and any lottery $\mathbf{x}$ with $\min (\mathbf{x}) \geq 0$, the relative gap between buying and selling price $\tau(W)$ is strictly decreasing in $W$.

Proof In the Appendix.

This proposition already gives an explanation of why the buying/selling price gap cannot be predicted within expected utility with consequentialism for small experimental lotteries. The reason is that within expected utility, the gap between buying and selling price is the highest for small values of wealth. So if initial wealth is small, the expected utility model can accommodate large buying and selling price gaps. 
Using Lemma 1 and Proposition 2.3 it is possible to infer certain properties of buying and selling price when data on relative gap between buying and selling price is available. Also, in the opposite direction, it is possible to infer properties of the relative gap between buying and selling price when certain properties of buying and selling price are known.

The above mathematical results can be best illustrated on the basis of an example. Let $\mathbf{x}$ be a lottery giving 100 euros or nothing with equal probabilities. The notation I use for such a lottery is $\left(100, \frac{1}{2} ; 0, \frac{1}{2}\right)$. Figure 1 contains graphs of selling and buying price for lottery $\mathbf{x}$ in the upper row and relative spread between them as functions of wealth $W$ in the lower row, each of them for CRRA utility function for three different coefficients of relative risk aversion: $1 / 2,1$ and $2^{4}$.

Notice that as stated in the propositions above, buying and selling prices are between $\min (\mathbf{x})$ and $\mathrm{E}[\mathbf{x}]$ for $\alpha=1$ and $\alpha=2$. For $\alpha=0.5$ I can calculate $W_{L}(\mathbf{x})$ as follows:

$$
W_{L}(\mathbf{x})=\left(\frac{1}{2} \sqrt{100}+\frac{1}{2} \sqrt{0}\right)^{2}=25
$$

Hence buying and selling price for $\alpha=0.5$ are indeed between $W_{L}(\mathbf{x})+\min (\mathbf{x})$ and $\mathrm{E}[\mathbf{x}]$. Notice also that buying and selling price are increasing and strictly concave in wealth and that selling price is higher than buying price over the whole domain of buying and selling prices. Finally as stated in Proposition 2.3 the relative gap indeed is the highest for the minimal value of wealth for which both buying and selling price are defined.

As illustrated by this simple example and stated formally in the propositions, the smaller the wealth the greater the relative gap between buying and selling price. So if wealth is small enough it is possible to obtain the gap between buying and selling price consistent with experimental evidence for reasonable levels of risk aversion. I will summarize this finding in a proposition.

Proposition 2.4 For levels of risk aversion which are consistent with experimental evidence on risk attitudes, there exists levels of wealth such that the expected utility model predicts a high relative gap between buying and selling price.

To make this finding more precise, notice that for the example above in the case of logarithmic utility function (the middle figure in Fig. 1), we have the relative gap between buying and selling price which is unbounded above as wealth tends to zero.

Furthermore, in order to obtain a selling price $30 \%$ higher than buying price for the lottery in consideration and for different relative risk aversion coefficients I need wealth levels which are listed in Table 1. For example to obtain a selling price $30 \%$ higher than buying price for the lottery $\left(100, \frac{1}{2} ; 0, \frac{1}{2}\right)$ for the logarithmic utility function, an initial wealth level of almost 44 is necessary. In the next

\footnotetext{
${ }^{4}$ The CRRA utility function is of the form given in Eq. 3.
} 

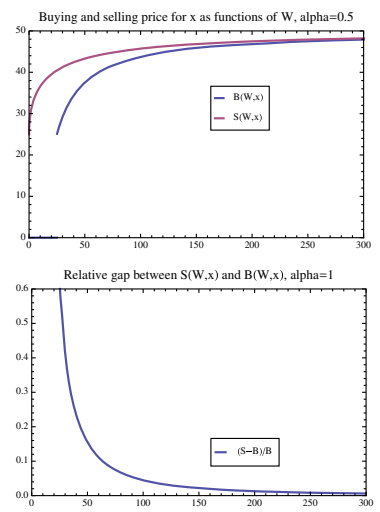
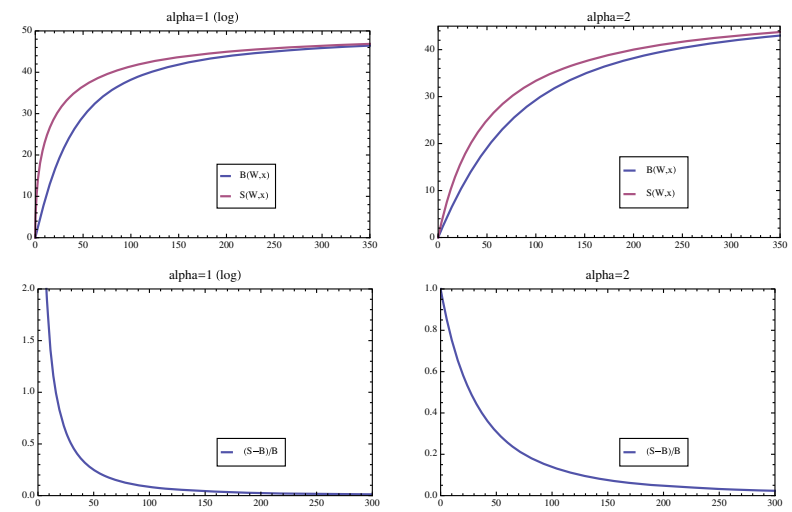

Fig. 1 Buying/selling price spread for $\mathbf{x}$ for CRRA utility function

subsection I introduce gambling wealth. If one believes that the expected utility model accurately predicts behavior, 44 would correspond to the calibrated gambling wealth.

Assuming that the decision maker exhibits constant relative risk aversion, one can calibrate pairs of wealth and relative risk aversion consistent with any given level of relative gap between selling and buying price for a given lottery.

\subsection{Expected utility with gambling wealth}

I have argued above that expected utility with total wealth interpretation of wealth predicts no gap between buying and selling price and risk neutrality for a wide range of gambles used in experiments. On the other hand I have shown that if small values of wealth are possible one can obtain large gaps between buying and selling price for a lottery for reasonable levels of risk aversion. One way to proceed would be to make wealth a free parameter of the model. Then, if one believes that expected utility is a good descriptive model of behavior, then given the data on risky choices one can calibrate which pairs of risk attitude and wealth level are consistent with the data, as I have illustrated in Table 1. Unfortunately, by making wealth a free parameter, the model loses much of its predictive power. In particular, it is harder to falsify the model or design testable predictions. Another way to proceed is to give wealth a new interpretation or, even better, to develop a theory of endogenous wealth determination and then to test whether this new interpretation gives better answers

Table 1 Selling price $30 \%$ higher than buying price

\begin{tabular}{llll}
\hline$\alpha$ & 0.5 & 1 & 2 \\
\hline$W$ & 35.15 & 43.94 & 51.57 \\
\hline
\end{tabular}


than consequentialist interpretation. Since at this point I am unable to offer a theory of endogenous wealth determination, I will only propose a new interpretation of wealth and ways to test it.

Gambling wealth Consequentialism assumption implies that when making any kind of decision, people consider and have in mind their lifetime wealth. I think a good alternative assumption is that people frame decisions narrowly and separate them into categories. When they engage themselves in housing decisions they think about a housing budget, when they consume they think about a consumption budget and when they consider gambling or whether to accept or reject an offered gamble, they consider a gambling budget. For the purposes of this paper I focus only on the gambling category and a budget assigned to it, which I call gambling wealth. Gambling wealth was proposed informally by Foster and Hart (2009). They define gambling wealth as that part of total wealth designated only for taking gambles. Alternatively, if $\underline{W}$ is wealth designated for the purposes of living, housing and consumption, then gambling wealth is what is left over.

In light of the results from the previous subsection, one can argue that the idea of gambling wealth, and more generally the idea of separate budgets for different categories of decisions, could explain a number of interesting phenomena, for example:

- Agents who gamble more have higher gambling wealth and therefore the buying and selling price gap for a given lottery is smaller than for less experienced individuals

- If an object is treated narrowly the disparity should be higher; if it is integrated into a wider set of objects the disparity should decrease (Hanemann 1991)

- Buying/selling price gap is more pronounced when objects of choice are not monetary, e.g. coffee mugs. The more specific or narrowly defined the object of choice is, the more pronounced wealth or income effects are.

The attractive feature of all these explanations is that they are all within the expected utility framework. The only novel thing is the narrow framing with which the expected utility model is supplemented. Naturally, a theory of endogenous wealth determination would be much appreciated to make this kind of explanation fully testable. At this point, however, it is necessary to design experiments to test the gambling wealth hypothesis. An example of a very simple such experiment is the following. In the first stage subjects are given a small amount of money for trading in gambles. Given different lotteries, buying price and selling price for them is elicited for each subject. In the second stage subjects are given more money for trading in gambles and again buying and selling price are elicited. Alternatively, instead of giving the subjects more money it is possible to scale down or up the lotteries being played. If subjects exhibit constant relative risk aversion it should be equivalent to increasing or decreasing initial wealth. If the gambling wealth explanation for the gap between buying and selling price is correct, then the gap should decrease when subjects are given more gambling money or if the lotteries are scaled down without changing gambling wealth. 
Assuming the approach is valid then I propose the following experiment for calibrating gambling wealth. The experiment should be designed to test risk attitudes ${ }^{5}$ and at the same time to elicit selling and buying prices for lotteries. Given the data it is then easy to calculate the underlying wealth level. This is then interpreted as gambling wealth. More precisely, given observed buying and selling price for a given lottery I can calculate a wealth-relative risk aversion coefficient pair which is consistent with these prices.

The gambling wealth hypothesis is promising. However, until there is no theory of endogenous gambling wealth determination, it cannot be fully testable. In the next subsection I discuss another concept which is related to gambling wealth-the concept of pocket cash by Fudenberg and Levine (2006). The advantage of the pocket cash idea is that there is a theory of pocket cash determination. I would like to show in what respect pocket cash and gambling wealth are similar and in what respect they differ.

Pocket cash The idea of pocket cash money in the context of gambling decisions is the following. If a small gamble is offered, an individual decides whether to take it or not on the basis of what he has in his pockets, and hence pocket cash will be the relevant wealth level for this decision. If, on the other hand, the same individual is offered a big gamble the values of which significantly exceed what he has in his pockets, the individual decides more carefully taking into account his lifetime wealth. I will introduce now some details of the model.

Fudenberg and Levine (2006) develop a dynamic model in which the long-run self controls the series of short-run selves. In each period $t$ there are two subperiods:

- bank subperiod

- consumption is not possible

- wealth $y_{t}$ is divided between savings $s_{t}$, which remain in the bank, and pocket cash $x_{t}$ which is carried to the nightclub

- nightclub subperiod

- consumption $0 \leq c_{t} \leq x_{t}$ is determined and $x_{t}-c_{t}$ is returned to the bank at the end of the period

- $\quad$ wealth next period is $y_{t+1}=R\left(s_{t}+x_{t}-c_{t}\right)$

The long-run self can implement $a^{*}$, the optimum of the problem without selfcontrol, by simply choosing pocket cash $x_{t}=\left(1-a^{*}\right) y_{t}$ to be the target consumption. In this way self-control costs might be avoided.

- At the nightclub in the first period there is a small probability the agent will be offered a choice between several lotteries.

- The model predicts then that:

- for large gambles risk aversion is relative to wealth

- for small gambles it is relative to pocket cash

\footnotetext{
${ }^{5}$ Characterization results from Lewandowski (2011) are useful here.
} 
In this way the model can explain (Rabin 2000)'s paradox and the large buying and selling price gap.

Gambling wealth vs. pocket cash An interesting feature of the Fudenberg and Levine (2006) approach is the following. Fudenberg and Levine (2006) estimate pocket cash to be roughly in the range of 20-100 dollars. This is very similar to the range of gambling wealth values, which are consistent with the experimental evidence on the buying/selling price gaps as indicated in Table 1. It is striking that the two entirely different approaches result in a very similar range of values for the underlying variable.

In spite of the similarities, the two concepts are nevertheless different from each other. To illustrate the difference I will now discuss what testable predictions are obtained in the Rabin (2000) paradox according to the dual self model with pocket cash and what testable predictions are obtained according to the gambling wealth approach.

Rabin (2000) calibrated that the expected utility model predicts the following:

- if a risk averse agent with wealth $\leq 350000$ rejects the lottery $(105,1 / 2 ;-100,1 / 2)$

- then he should reject the lottery $(635000,1 / 2 ;-4000,1 / 2)$ at wealth level 340000

Denote the first of the above lotteries by lottery 1 and the second by lottery 2 . According to Rabin (2000) the first statement is plausible and the second is not and hence it is called a paradox.

In the dual-self model it is not true anymore that the decision maker rejects both lotteries. The first lottery is small and hence it is evaluated relative to pocket cash. The second lottery is big and therefore it is evaluated according to total wealth. Suppose that the utility function is logarithmic. Then the following is true:

- $\quad$ lottery 1 small - reject if pocket cash $<2100$

- lottery 2 large - accept if total wealth higher than 4035

Now both statements (pocket cash less than 2100 and total wealth higher than 4035) are plausible.

Now consider the gambling wealth interpretation. Suppose that utility is logarithmic. If gambling wealth is less than 2100 then the decision maker should

- $\quad$ reject lottery 1

- reject lottery 2

There is nothing paradoxical in rejecting the second lottery since gambling wealth in the amount of 2100 is too little to cover the loss $(-4000)$ which occurs with probability $1 / 2$. No matter how attractive the second prize is, the decision maker cannot afford to take lottery 2 .

Rabin's paradox in the literature Although, in this paper, I adopt the lines of Rubinstein (2006), and focus on the assumption of consequentialism, there have 
been other explanations for Rabin's paradox in the literature. Palacios-Huerta and Serrano (2006) claim that it is the assumption of rejecting small gambles over a large range of wealth levels, which should be questioned as it does not match realworld behavior. In particular they show that the assumption that an expected utility maximizer turns down a given even-odds gamble with gain and loss for a given range of wealth levels implies that there exists a positive lower bound on the coefficient of absolute risk aversion which can be calculated exactly. This lower bound is an additional assumption imposed on a utility function. Palacios-Huerta and Serrano (2006) show that in Rabin's examples this lower bound turns out to be very high, which is not consistent with empirical evidence. Another paper which addresses Rabin's critique of expected utility is Cox and Sadiraj (2006). In a similar spirit to Rubinstein (2006), they claim that Rabin in fact criticizes the expected utility model of terminal wealth, in which there is a single preference relation over final wealth consequences. They show that the expected utility of income model, in which prizes are interpreted as changes in wealth levels, does not exhibit Rabin's paradoxical behavior. In order to enable the dependence of preference over income on initial wealth, they design an expected utility of initial wealth and income model. They demonstrate that such a model can withstand Rabin's critique if initial wealth is not additive to income in the utility function. Safra and Segal (2008) on the other hand point out that paradoxes of the kind considered by Rabin are not specific to expected utility theory. They show that they can be constructed in non-expected utility theories as well.

\section{Preference reversal versus buying/selling price reversal}

Preference reversal is commonly observed in experiments. Suppose that $A \succ_{C} B$ denotes "A preferred to B in a direct choice". Using my notation, preference reversal is possible if:

$$
S(W, \mathbf{y})>S(W, \mathbf{x}) \text { and } \mathbf{x} \succ_{C} \mathbf{y}
$$

Preference reversal is not possible within the expected utility framework. To see this, note that expected utility implies that $\mathbf{x} \succ_{C} \mathbf{y}$ which can be equivalently written as $\mathrm{E} U(W+\mathbf{x})>\mathrm{E} U(W+\mathbf{y})$. By definition of $S$, this is equivalent to $U(W+$ $S(W, \mathbf{x}))>U(W+S(W, \mathbf{x}))$ and since the utility function is strictly increasing: $S(W, \mathbf{x})>S(W, \mathbf{y})$. So expected utility implies the following:

$$
S(W, \mathbf{y})>S(W, \mathbf{x}) \Longleftrightarrow \mathbf{y} \succ_{C} \mathbf{x}
$$

On the other hand the buying/selling price reversal is possible within the expected utility framework:

Proposition 3.1 For a given decreasing absolute risk aversion utility function and any wealth level $W$, buying/selling price reversal is possible.

Proof In the Appendix. 
By condition (5) this proposition implies that expected utility admits the possibility of the following kind of preference reversal:

$$
B(W, \mathbf{y})>B(W, \mathbf{x}) \text { and } \mathbf{x} \succ_{C} \mathbf{y}
$$

This kind of preference reversal will be referred to as preference reversal B. Preference reversal B is equivalent to buying/selling price reversal within the expected utility framework.

Since expected utility theory imposes rather strong consistency assumptions, the result above suggests that the possibility of preference reversal is less rational than the related possibility of buying/selling price reversal or preference reversal B. The following two propositions clarify the meaning of "less rational" beyond the strength of consistency requirements argument.

Proposition 3.2 Suppose that preferences of the decision maker are continuous, monotonic and that the preference reversal pattern is fixed for the range of wealth $W \in[\underline{w}, \bar{w}]$. Then arbitrage opportunities exist.

Proof In the Appendix.

Hence preference reversal allows arbitrage. On the other hand buying/selling price reversal or preference reversal B does not allow arbitrage.

Proposition 3.3 Buying/selling price reversal does not allow arbitrage.

Proof In the Appendix.

The analysis shows that buying/selling price reversal, or preference reversal B, is more rational than traditional preference reversal in two respects-it is consistent with expected utility and it does not allow arbitrage.

Preference reversal B or buying/selling price reversals occur within expected utility theory. However it does not mean that they have to be meaningful. If buying/selling price gap is small, then these two reversals are not meaningful i.e. they can occur theoretically but the scope for their occurrence is negligible. For these reversals to be meaningful, it is necessary for buying/selling price gap to be non-negligible. Testing of preference reversal B might be therefore relevant only if wealth is interpreted narrowly, either as gambling wealth or pocket cash. It is not relevant if the doctrine of consequentialism is maintained. I will illustrate this fact in the following example.

Example 1 Suppose the utility function is CRRA with relative risk aversion coefficient of 2 , the $\$$-bet (denote it by $\mathbf{x}$ ) gives $\$ 100$ or $\$ 0$ with equal probabilities and the P-bet (denote it by $\mathbf{y}$ ) gives $\$ 40$ with probability $3 / 4$ and $\$ 0$ otherwise. Figure 2 contains graphs of buying and selling prices for these two lotteries as functions of wealth. 


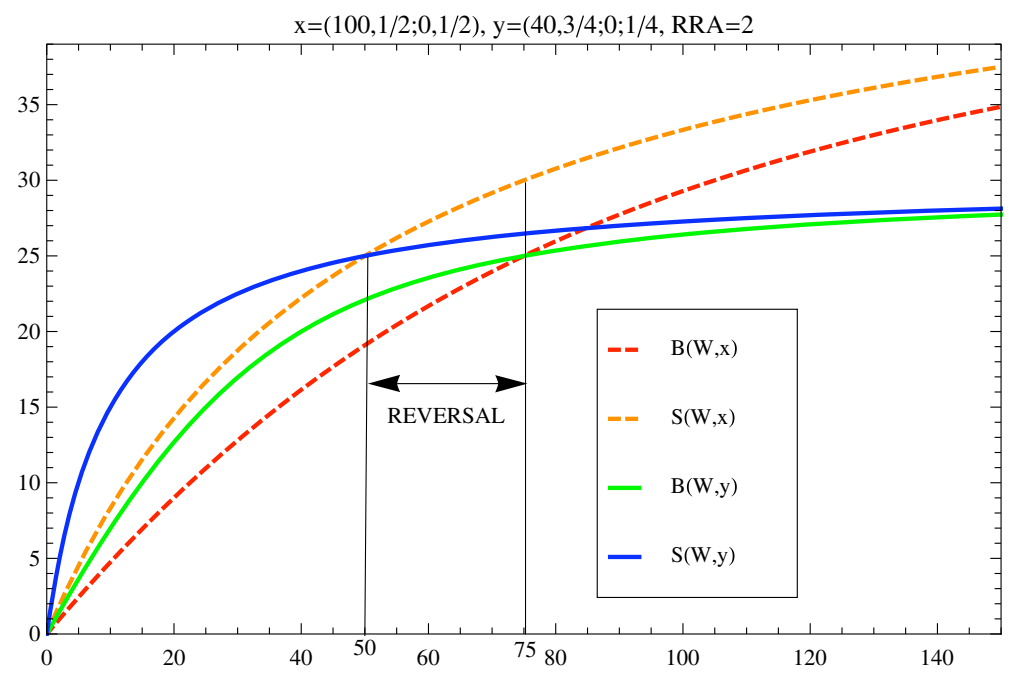

Fig. 2 Wealth region for buying/selling price reversal

In the above example, there is an interval $(50,75)$ of wealth for which buying/selling price reversal (and hence also preference reversal B) occurs ${ }^{6}$. This is the common pattern that buying/selling price reversal occurs only at small wealth and only in the limited interval of wealth. The reason is that for such reversal to occur the \$-bet has to have higher variance and higher expected value. Then as wealth becomes large and the buying and selling price approach the expected value of a lottery, these prices for the \$-bet have to increase above those of the P-bet. For smaller values of wealth, the CRRA decision maker would become very risk averse, so he will be solely preoccupied by the gamble's variance. Therefore, both selling and buying price for the $\$$-bet are below those of the P-bet. Technically speaking, notice that if $W^{*}$ denotes the wealth level at which selling price of $\mathbf{x}$ and $\mathbf{y}$ are equal, i.e. $S\left(W^{*}, \mathbf{x}\right)=S\left(W^{*}, \mathbf{y}\right)=S^{*}$, then from definition it also holds that $S^{*}=B\left(W^{*}+S^{*}, \mathbf{x}\right)=B\left(W^{*}+S^{*}, \mathbf{y}\right)$, so that $B(W, \mathbf{x}) \operatorname{crosses} B(W, \mathbf{y})$ at $W=W^{*}+S^{*}$. Hence the interval for which buying/selling price reversal occurs is of length $S^{*}$ exactly.

\section{Discussion on consequentialism}

A typical behavioral paper first presents or discusses experimental evidence which is not consistent with rationality assumptions of expected utility. Afterwards it introduces or discusses a behavioral model capable of accommodating presented evidence. When we talk about the inconsistency of a traditional theory with new

${ }^{6}$ The following holds $S(50, \mathbf{y})=S(50, \mathbf{x})$ and $B(75, \mathbf{y})=B(75, \mathbf{x})$. 
experimental evidence, it is important to identify what the term "traditional theory" means exactly. Rubinstein (2006) claims that a lot of recent confusion around expected utility, which led some researchers to question it as a descriptive theory, is caused by associating expected utility theory with the assumption of consequentialism. Consequentialism is the idea that there is a single preference relation $\succsim$ over the set of lotteries with prizes being the "final wealth levels" such that the decision maker at any wealth level $W$ who has a von Neumann Morgenstern preference relation $\succsim W$ over the set of "wealth changes" derives that preference from $\succsim$ by:

$$
L_{1} \succsim_{W} L_{2} \Longleftrightarrow W+L_{1} \succsim W+L_{2}
$$

where $L_{1}$ and $L_{2}$ are lotteries. Also, Cox and Sadiraj (2006) argue that the confusion around expected utility is caused by the failure in the literature to distinguish between expected utility theories, which stands for all models based on a set of axioms among which there is the independence axiom, and a specific expected utility model. A similar argument is also given in Palacios-Huerta and Serrano (2006) ${ }^{7}$. Based on the above references, a clear distinction should be made between expected utility theory, which is an abstract mathematical theory developed by von Neumann and Morgenstern, and a specific expected utility model. Abstract mathematical theory should be judged on different grounds than specific economic models of decisionmaking which assume certain interpretation to the objects of choice, preference relations, etc. For example von Neumann Morgenstern theory is silent about the identity of prizes in any given lottery. Hence, defining lottery prizes as being final wealth levels (often assumed in expected utility models) or defining lottery prizes as being changes relative to a reference point (as in prospect theory) is not part of expected utility theory ${ }^{8}$.

As a result, seeking interpretation for abstract mathematical objects necessitates imposing additional behavioral assumptions. Based on the specific interpretation imposed we will classify expected utility models accordingly into:

- Traditional expected utility models

- Expected utility assuming consequentialism (Usually identified with "expected utility" by much of the behavioral literature.)

- Expected utility with gambling wealth ${ }^{9}$

- Expected utility of wealth changes ${ }^{10}$

- Behavioral expected utility models

- Prospect Theory without probability weighting (only reference dependence)

\footnotetext{
${ }^{7}$ Many of the aforementioned references focused their discussion around the Rabin (2000) paradox, but these arguments are more general and refer to the whole debate about expected utility and behavioral theories.

${ }^{8}$ This example shows that reference dependence in prospect theory is not a departure from expected utility theory as a mathematical theory. On the other, probability weighting, which is the second building block of prospect theory, clearly constitutes a departure from expected utility theory, because irrespective of the interpretation, nonlinearity in the objectively given probabilities violates the independence assumption of expected utility theory.
} 
- Expected disappointment model (Gul 1991)

- Regret theory (Loomes and Sugden 1982; Bell 1982)

Below I will try to illustrate the difference between these theories, limiting myself to only the difference which is the basis for the above classification. Suppose there is a lottery: with equal chances to win $\$ 12$ or lose $\$ 10$.

- Traditional expected utility models

- Expected utility assuming consequentialism

* Lottery payoffs defined as total wealth positions

* Single preference relation over lifetime wealth: e.g. $\$ 10 \mathrm{mln}$.

* Accept if (\$10 000 012, 0.5; $9 \$ 999990,0.5) \succsim \$ 10000000$

* Problem: (Rabin 2000) paradox

- EU with gambling wealth

* Treat decisions narrowly, reinterpret $W$, e.g. gambling wealth instead of total wealth

* $\quad$ Accept if $(\$ 112,0.5 ; \$ 90,0.5) \succsim \$ 100$

* E.g. relative changes (returns) and log utility function ${ }^{11}$

* Accept because $1.12 \times 0.9>1$

- EU of wealth changes

* $\quad$ Accept if $(\$ 12,0.5 ;-\$ 10,0.5) \succsim \$ 0$

* E.g. nominal changes and CARA utility function

* Examples: Game theory, Auction theory

- Behavioral expected utility models

- Prospect Theory without probability weighting

* The same as EU of wealth changes + loss aversion, reflection effect, etc.

* If reference point current wealth then accept if $(\$ 12,0.5 ;-\$ 10,0.5) \succsim \$ 0$

- Model of disappointment aversion

* Reference point: current wealth $+E(L)$

* Accept if $(\$ 11,0.5 ;-\$ 11,0.5) \succsim-\$ 1$

- Regret theory

* Reference point: other lottery

- Non-EU models

- Non-EU models are not linear in probabilities

\footnotetext{
${ }^{9}$ The term introduced in Foster and Hart (2009) and adopted throughout this paper.

${ }^{10}$ Used in general in much of game theory and in particular in the auction literature.
} 
- Examples:

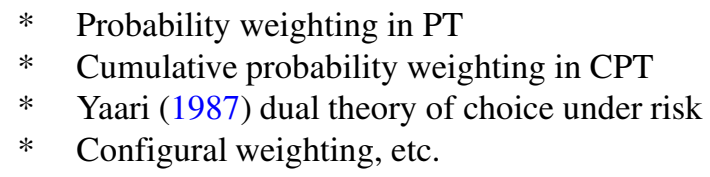

\section{Expected utility over wealth changes}

I argued above that dropping the assumption of consequentialism and interpreting initial wealth narrowly as something much smaller than total wealth can explain large buying and selling price gaps but cannot explain traditional preference reversal involving WTA valuations. But in my approach, the consequentialist view, while dropped, is replaced with something different only in quantitative and not qualitative terms. I allow initial wealth to be small enough with a claim that it changes the interpretation of initial wealth. This approach changes things only slightly in the following sense: While in the case of total wealth interpretation of initial wealth, an individual's preferences over wealth changes are induced from his preferences over final wealth levels, in the case of gambling wealth, preferences over changes in gambling wealth are induced from preferences over gambling wealth levels. In quantitative terms, these two situations may be very different. However in a structural or qualitative sense, these two situations differ only marginally. A much more fundamental departure from the consequentialism assumption would be the following-suppose that the decision maker derives preference over lotteries not from preferences over the resulting total position of whatever budget he might consider, but directly from changes to this budget implied by accepting the lottery in question. This approach was first undertaken by Kahneman and Tversky (1979) in their seminal contribution. In prospect theory, which was then proposed as an alternative to expected utility theory, the decision maker has preferences directly over wealth changes relative to a reference point. Rubinstein (2006) suggests that a similar approach is possible without having to depart from expected utility theory by reinterpreting lottery prizes as monetary change and not as total position. In what follows I will illustrate formally that in such an approach both a large buying and selling price gap and preference reversal are possible for a wide class of utility functions defined over wealth changes.

\subsection{WTA/WTP disparity for preferences over wealth changes}

Suppose $U(\cdot)$ is a utility function defined over changes in wealth, and hence over the whole real line. It is assumed to be strictly increasing, continuous and that $U(0)=0$. Willingness to accept for lottery $\mathbf{x}$, denoted by $W T A(\mathbf{x})$, and willingness to pay for

\footnotetext{
${ }^{11}$ Notice that reference dependence is not a novel feature of Prospect Theory, but it exists in expected utility models when prizes are interpreted as returns-relative reference dependence compared to nominal reference dependence in Prospect Theory.
} 
lottery $\mathbf{x}$, denoted by $W T P(\mathbf{x})$, will be used here in place of $S(W, \mathbf{x})$ and $B(W, \mathbf{x})$, respectively. They are defined as follows:

$$
\begin{aligned}
& \mathrm{E} U(W T A(\mathbf{x})-\mathbf{x})=0 \\
& \mathrm{E} U(\mathbf{x}-W T P(\mathbf{x}))=0
\end{aligned}
$$

The meaning of these two measures is equivalent to the meaning of $S(W, \mathbf{x})$ and $B(W, \mathbf{x})$ for the case of expected utility of wealth changes model.

Proposition 5.1 Suppose that $U(x)<-U(-x)$ for all $x>0$. Then given any nondegenerate lottery $\mathbf{x}$, the following holds: WT $A(\mathbf{x})>W T P(\mathbf{x})$.

Proof Denote $A \equiv W T A(\mathbf{x})$ to save on notation.

$$
\begin{aligned}
\mathrm{E}[U & (\mathbf{x}-A)-U(A-\mathbf{x})] \\
& =\sum_{i: x_{i}>A} p_{i}\left[U\left(x_{i}-A\right)-U\left(-\left(x_{i}-A\right)\right)\right]-\sum_{i: x_{i} \leq A} p_{i}\left[U\left(A-x_{i}\right)-U\left(-\left(A-x_{i}\right)\right)\right] \\
& <-2 \sum_{i: x_{i}>A} p_{i} U\left(-\left(x_{i}-A\right)\right)-2 \sum_{i: x_{i} \leq A} p_{i} U\left(A-x_{i}\right) \\
& =0
\end{aligned}
$$

The claim follows by monotonicity of $U$.

The above proposition is quite general. In what follows I will analyze two special cases. In the original version of prospect theory (Kahneman and Tversky 1979) the reference point was required to be constant. Hence it would be impossible to define willingness to accept in this formulation. Schmidt et al. (2008) proposed a so called "third-generation prospect theory" in which the reference point is allowed to be random. They defined willingness to accept and willingness to pay essentially as in Eqs. 6 and 7 and showed first that a willingness to pay and willingness to accept disparity is possible in prospect theory mainly due to loss aversion. Below I will show my version of their result which shows that under certain symmetry conditions the WTA/WTP gap occurs solely due to loss aversion. Consider the prospect theory utility function with an imposed symmetry condition of the following form:

Assumption 2 Utility function for outcomes is of the following form:

$$
U(x)=\left\{\begin{array}{cl}
u(x) & \text { if } x \geq 0 \\
-\lambda u(-x) & \text { if } x<0
\end{array}\right.
$$

where $\lambda>0$ denotes the loss attitude parameter. If $\lambda>1$, there is loss aversion. Furthermore, a function $u(\cdot)$ is absolutely continuous, bounded, strictly increasing with $u(0)=0$ and concave on its domain.

The above utility function is concave for gains, convex for losses and for $\lambda=1$, it is symmetric around $(0,0)$, meaning that risk loving for losses is of the same magnitude as risk aversion for gains. For $\lambda>1$, there is loss aversion, which means that a given gain brings less satisfaction than the dissatisfaction from the same loss. 
Observe that this utility function satisfies $U(x)<-U(-x)$ for all $x>0$ if and only if $\lambda>1$. Hence for $\lambda$ greater than one, meaning that there is loss aversion, willingness to accept for any nondegenerate lottery exceeds willingness to pay by Proposition 5.1. In fact for a utility function of the above form an even stronger result holds, which I state below:

Proposition 5.2 For a nondegenerate lottery $\mathbf{x}$ and utility function of the form defined by Assumption 2, the following holds:

$$
\lambda>1 \Longleftrightarrow W T A(\mathbf{x})>W T P(\mathbf{x})
$$

Proof Define $A \equiv W T A(\mathbf{x})$ and $P \equiv W T P(\mathbf{x})$ to save on notation. From definitions:

$$
\begin{aligned}
\lambda \sum_{i: x_{i}>A} u\left(x_{i}-A\right) & =\sum_{i: x_{i} \leq A} u\left(A-x_{i}\right) \\
\sum_{i: x_{i}>P} u\left(x_{i}-P\right) & =\lambda \sum_{i: x_{i} \leq P} u\left(P-x_{i}\right)
\end{aligned}
$$

First notice that $\lambda=1$ if and only if $A$ is equal to $P$. Now observe that $\lambda>1$ if and only if

$$
\sum_{i: x_{i}>A} u\left(x_{i}-A\right)<\lambda \sum_{i: x_{i}>A} u\left(x_{i}-A\right)=\sum_{i: x_{i} \leq A} u\left(A-x_{i}\right)<\lambda \sum_{i: x_{i} \leq A} u\left(A-x_{i}\right)
$$

And by monotonicity of $u$ it follows immediately that $A>P$ and hence $W T A(\mathbf{x})>$ $W T P(\mathbf{x})$.

The above proposition shows that in prospect theory, a willingness to accept/willingness to pay disparity may be explained solely by loss aversion.

An even simpler version of this result obtains in case of the prospect theory utility function without risk aversion.

Assumption 3 Utility function for outcomes is of the following form:

$$
U(x)=\left\{\begin{array}{cc}
x & \text { if } x \geq 0 \\
\lambda x & \text { if } x<0
\end{array}\right.
$$

Proposition 5.3 For a nondegenerate lottery $\mathbf{x}$ and utility function defined by Assumption 3

$$
\lambda>1 \Longleftrightarrow W T A(\mathbf{x})>\mathrm{E}[\mathbf{x}]>W T P(\mathbf{x})
$$


Proof Define $A \equiv W T A(\mathbf{x})$ to save on notation. From definition:

$$
\begin{aligned}
0 & =-\lambda \sum_{i: x_{i}>A} p_{i}\left(x_{i}-A\right)+\sum_{i: x_{i} \leq A} p_{i}\left(A-x_{i}\right) \\
& =(1-\lambda) \sum_{i: x_{i}>A} p_{i}\left(x_{i}-A\right)+\sum_{i=1}^{n} p_{i}\left(A-x_{i}\right) \\
& =(1-\lambda) \sum_{i: x_{i}>A} p_{i}\left(x_{i}-A\right)+A-\mathrm{E}[\mathbf{x}] \\
& <A-\mathrm{E}[\mathbf{x}]
\end{aligned}
$$

The proof that $\mathrm{E}[\mathbf{x}]>W T P(\mathbf{x})$ is similar and hence omitted.

In fact utility function defined by Assumption 3 is a special case of an overall concave utility function for which a similar result holds:

Proposition 5.4 For a nondegenerate lottery $\mathbf{x}$, given utility function $u: \mathbb{R} \rightarrow \mathbb{R}$ that is strictly increasing, continuous and bounded with $u(0)=0$, the following holds:

$$
u(\cdot) \text { is concave } \Longleftrightarrow W T A(\mathbf{x}) \geq \mathrm{E}[\mathbf{x}] \geq W T P(\mathbf{x})
$$

Proof By Jensen's inequality:

$$
\begin{aligned}
& 0=\mathrm{E} u(\mathbf{x}-W T P(\mathbf{x})) \leq u(\mathrm{E}[\mathbf{x}]-W T P(\mathbf{x})) \\
& 0=\mathrm{E} u(W T A(\mathbf{x})-\mathbf{x}) \leq u(W T A(\mathbf{x})-\mathrm{E}[\mathbf{x}])
\end{aligned}
$$

Since $u(0)=0$ and $u$ is strictly increasing, the conclusion follows.

The conclusion of this section is that the gap between willingness to accept and willingness to pay in the case of preferences defined over wealth changes and not wealth levels may be explained by a kind of a general loss aversion, which is defined by the requirement: $u(x)<-u(-x)$, for all $x>0$. This requirement defines a wide class of available utility functions and in particular, an $\mathrm{S}$ shaped utility function with sufficient level of loss aversion as well as a traditional overall concave utility function over the whole real line satisfies this condition.

\subsection{Preference reversal for preferences over wealth changes}

First, recall that traditional preference reversal is not possible within expected utility when preferences are defined over wealth levels, irrespective of whether these wealth levels are interpreted narrowly as levels of gambling wealth, for instance, or whether they are interpreted traditionally as total wealth levels. On the other hand, when preferences are defined over wealth changes, it turns out that traditional preference reversal is possible. Schmidt et al. (2008) shows that preference reversal may occur in third generation prospect theory. They calibrate for which values of parameters a quite general (and yet parametrized) version of prospect theory is compatible 
with preference reversal. Below I will show that for a very simple version of third generation prospect theory, preference reversal is obtained as a generic element.

Let $\mathbf{x} \equiv(x, p)$ and $\mathbf{y} \equiv(y, q)$ be two prospects such that $y>x>0$ and $1>p>q>0$. Lottery $\mathbf{x}$ will be called the P-bet and lottery $\mathbf{y}$ will be called the $\$$-bet. In what follows I want to demonstrate that preference reversal is possible.

Lemma 3 For a utility function satisfying Assumption 3, the following holds: If $p x=q y$, so that the decision maker is indifferent between lottery $\mathbf{x}$ and $\mathbf{y}$ in a direct choice, then

$$
\lambda>1 \Longleftrightarrow W T A(\mathbf{y})>W T A(\mathbf{x})
$$

Proof From definitions I calculate that:

$$
W T A(\mathbf{x})=\frac{\lambda p}{1-p+\lambda p} x, \quad W T A(\mathbf{y})=\frac{\lambda q}{1-q+\lambda q} y
$$

Using the fact that $p x=q y$, I obtain:

$$
W T A(\mathbf{y})-W T A(\mathbf{x})=W T A(\mathbf{y}) \frac{p-q}{1-p+\lambda p}(\lambda-1)
$$

The claim immediately follows.

Proposition 5.5 Given a utility function satisfying Assumption 3, preference reversal is possible if and only if $\lambda>1$

Proof Preference reversal occurs when the decision maker chooses the P-bet in a direct choice but assigns higher willingness to accept to the \$-bet. In terms of the model, preference reversal occurs when $p x>q y$ and $W T A(\mathbf{y})>W T A(\mathbf{x})$. By Lemma 3, I know that if $p x=q y$ then $W T A(\mathbf{y})>W T A(\mathbf{x}) \Longleftrightarrow \lambda>1$. Since utility function $u(\cdot)$ is continuous, it follows that willingness to accept as a function of a given lottery is also continuous. Hence changing the lottery slightly changes willingness to accept for it slightly. It follows that if initially $p x=q y$ and I increase $p$ or $x$ slightly or decrease $q$ or $y$ slightly, the new lottery $\mathbf{x}$ will be preferred to a new lottery $\mathbf{y}$ in a direct choice and yet it will remain true that willingness to accept for a new lottery $\mathbf{y}$ will still be higher than willingness to accept for a new lottery $\mathbf{x}$.

Again, a more general result for concave functions is possible:

Lemma 4 Suppose that $p x=q y$. Given utility function $u: \mathbb{R} \rightarrow \mathbb{R}$ that is strictly increasing, continuous and bounded with $u(0)=0$, the following holds:

$$
u(\cdot) \text { is concave } \Longleftrightarrow W T A(\mathbf{y}) \geq W T A(\mathbf{x})
$$


Proof Define $A \equiv W T A(\mathbf{x})$ to save on notation. A satisfies the following equation:

$$
p u(A-x)+(1-p) u(A)=0
$$

The following classical result, called the three-strings lemma, is needed for the proof:

Lemma 5 (Three strings lemma) Utility function $u(\cdot)$ is concave if and only if for $a>b>c$ the following holds:

$$
\frac{u(a)}{a}<\frac{u(b)}{b}<\frac{u(c)}{c}
$$

Hence $u(\cdot)$ is concave if and only if

$$
\begin{aligned}
q u(A-y)+(1-q) u(A) & \leq q \frac{A-y}{A-x} u(A-x)+(1-q) u(A) \\
& =u(A-x)\left[q \frac{A-y}{A-x}-p\right]+(p-q) u(A) \\
& =u(A-x) \frac{q A-q y-p A+p x}{A-x}+(p-q) u(A) \\
& =A(p-q)\left[\frac{u(A)}{A}-\frac{u(A-x)}{A-x}\right] \\
& \leq 0
\end{aligned}
$$

Proposition 5.6 Suppose that $p x=q y$. Preference reversal occurs if $u(\cdot)^{12}$ is strictly concave.

Proof Suppose that $u(\cdot)$ is strictly concave. By Lemma 4, WTA(y) $>W T A(\mathbf{x})$. Since $x<y, \frac{u(x)}{x}>\frac{u(y)}{y}$, by the three strings lemma for concave function $u(\cdot)$. Hence the following holds:

$$
\mathrm{E} u(\mathbf{x})=p u(x)>p \frac{x}{y} u(y)=q u(y)=\mathrm{E} u(\mathbf{y})
$$

So lottery $\mathbf{x}$ or a P-bet is chosen over lottery $\mathbf{y}$ or a $\$$-bet in a direct choice and yet $W T A(\mathbf{y})>W T A(\mathbf{x})$ as required.

Concavity of a utility function is sufficient for preference reversal in the above example. However it is not necessary. In particular, Schmidt et al. (2008) show that preference reversal is possible with an S-shaped prospect utility function, which is convex for losses. If one wants to obtain a possibility of preference reversal for specific lotteries and not as a generic feature of the model, then the following requirement, which is weaker than the overall concavity of the utility function, may

\footnotetext{
${ }^{12}$ Strictly increasing, continuous and $u(0)=0$.
} 
be imposed: For a given P-bet and a given \$-bet and utility function $u(\cdot)$, define $A=W T A(\mathbf{x})$. Then:

$$
\frac{u(A-y)}{A-y}>\frac{u(A-x)}{A-x}>\frac{u(A)}{A}
$$

\section{Concluding remarks}

Expected utility theory by von Neumann and Morgenstern (1944) imposes a set of consistency assumptions on choices among lotteries. The theory is used in a large part of economic theory, including the famous Nash existence theorem. However there is a lot of mainly experimental evidence that people often violate von Neumann and Morgenstern (1944) axioms, in particular the most crucial among them-independence. In response to this evidence economists started to question expected utility theory and investigate other models of choice which describe human behavior better. However, since these new theories usually have lower consistency requirements being imposed on the admissible choice, they necessarily also have lower prediction power and less scope for testable predictions. Moreover, they also have weaker normative appeal, since the decision makers violating expected utility axioms are vulnerable to money pumps. It is therefore an important issue to identify patterns of choices and behavior which are consistent with expected utility and contrast them with those which are impossible within expected utility. In order to perform this task it is important to identify expected utility theory in its bare form and in particular separate it from the doctrine of consequentialism. More precisely, it is necessary to abandon the common practice of interpreting the wealth variable as total wealth position common to all decisions.

If one is willing to accept that wealth underlying gambling decisions is separated from total wealth so that gambling decisions are framed narrowly, important implications can be derived. If gambling wealth is small enough, which should be tested in an experiment, then selling price for a lottery can be significantly greater than buying price without going beyond the expected utility model, and the extent of this difference can be as high as the one found in experiments. Also, the famous (Rabin 2000) paradox can be resolved, suggesting that expected utility is not guilty here, but rather the doctrine of consequentialism.

Still, traditional preference reversal is not possible even if wealth is allowed to be small. If expected utility is to be regarded as a positive theory, it is definitely a negative result. However, if one is willing to accept expected utility as a good normative theory, then the same result is very useful. It informs us then that preference reversal is not rational. It is confirmed further by the result proved in the paper, that individuals exhibiting preference reversal are susceptible to arbitrage under certain mild conditions. The same kind of arbitrage, which I prefer to call strong arbitrage, is not possible within expected utility. What might be interesting is that another kind of preference reversal, which I call preference reversal B and which involves buying price in place of selling price and otherwise is the same as the traditional 
preference reversal, is possible within expected utility and is not vulnerable to arbitrage as shown in the paper.

Acknowledgments I would like to thank Pascal Courty, Fernando Vega-Redondo, Roberto Serrano, Robert Sugden, David Levine, John Sutton, Kip Viscusi and audiences of many seminars for help, support, advice and guidance.

Open Access This article is distributed under the terms of the Creative Commons Attribution License which permits any use, distribution, and reproduction in any medium, provided the original author(s) and the source are credited.

\section{Appendix}

In what follows I will need the following lemma:

Lemma 6 For any lottery $\mathbf{x}$ and any wealth level $W$, the following holds:

$$
\begin{aligned}
& S[W, \mathbf{x}-B(W, \mathbf{x})]=0 \\
& S[W-B(W, \mathbf{x}), \mathbf{x}]=B(W, \mathbf{x}) \\
& B[W+S(W, \mathbf{x}), \mathbf{x}]=S(W, \mathbf{x})
\end{aligned}
$$

The proof is directly from definitions. For details, see Lewandowski (2011).

\section{Proof of Proposition 1.1}

Notice first, that for degenerate lottery $\mathbf{x}=x$, Eqs. 1 and 2 imply $S(W, x)=$ $B(W, x)=x$. From now on I will focus on a non-degenerate lottery $\mathbf{x}$. I will prove the proposition only for the case of selling price. For buying price the proof is similar. I define $S \equiv S(W, \mathbf{x})$. Suppose $\min _{i \in\{1, \ldots, n\}} x_{i} \geq S$. Then notice that: $U\left(W+x_{i}\right) \geq U\left(W+\min _{i \in\{1, \ldots, n\}} x_{i}\right) \geq U(W+S)$ with strict inequality for any $x_{i} \neq \min _{i \in\{1, \ldots, n\}} x_{i}$. Since lottery $\mathbf{x}$ is non-degenerate there exists at least one $x_{i} \neq \min _{i \in\{1, \ldots, n\}} x_{i}$ Hence $\sum_{i=1}^{n} p_{i} U\left(W+x_{i}\right)>U(W+S)$ So $S$ cannot be the selling price-a contradiction.

Suppose now that $S \geq \mathrm{E}[\mathbf{x}]$. By strict Jensen's inequality $\mathrm{E} U[W+\mathbf{x}]<U[W+$ $\mathrm{E}[\mathbf{x}]] \leq U(W+S)$ So $S$ cannot be the selling price-a contradiction. So I have shown that indeed $\min _{i \in\{1, \ldots, n\}} x_{i}<S(W, \mathbf{x})<\mathrm{E}[\mathbf{x}]$.

\section{Proof of Proposition 2.1}

Note first that $U_{\alpha}$ is unbounded from below if $\alpha \geq 1$ and bounded from below if $\alpha<1$.

$$
\lim _{x \rightarrow 0} U_{\alpha}(x)=\left\{\begin{array}{lr}
-\frac{1}{1-\alpha}, & 0<\alpha<1 \\
-\infty, & \alpha \geq 1
\end{array}\right.
$$

By Proposition 1.1 buying and selling prices are necessarily greater than $\min (\mathbf{x})$. For $\alpha \geq 1$ the utility function is unbounded from below, therefore from the definition 
it follows that: $\lim _{W \rightarrow 0} B(W, \mathbf{x})=\min (\mathbf{x})$ and $\lim _{W \rightarrow-\min (\mathbf{x})} S(W, \mathbf{x})=\min (\mathbf{x})$. On the other hand for $0<\alpha<1$ the utility function is bounded from below. Additionally, $W-B(W, \mathbf{x})$ is strictly increasing in $W$ since $\frac{\partial B(W, \mathbf{x})}{\partial W}<1$. Therefore the lower bound for the domain of $B(W, \mathbf{x})$ as a function of $W$ is given by $W_{L}(\mathbf{x})$ such that: $\mathrm{E} U(-\min (\mathbf{x})+\mathbf{x})=U\left(W_{L}(\mathbf{x})\right)$. It follows that $\lim _{W \rightarrow W_{L}(\mathbf{x})} B(W, \mathbf{x})=$ $W_{L}(\mathbf{x})+\min (\mathbf{x})$. Similarly, the lower bound for the domain of $S(W, \mathbf{x})$ as a function of $W$ is $-\min (\mathbf{x})$ and hence: $\lim _{W \rightarrow-\min (\mathbf{x})} S(W, \mathbf{x})=W_{L}(\mathbf{x})+\min (\mathbf{x})$ since $\mathrm{E} U(-\min (\mathbf{x})+\mathbf{x})=U(-\min (\mathbf{x})+S(-\min (\mathbf{x}), \mathbf{x}))=U(-\min (\mathbf{x})+\min (\mathbf{x})+$ $\left.U^{-1}(\mathrm{E} U(-\min (\mathbf{x})+\mathbf{x}))\right)$

Now I prove the following statement: $\forall \alpha>0, \lim _{W \rightarrow \infty} B(W, \mathbf{x})=$ $\lim _{W \rightarrow \infty} S(W, \mathbf{x})=\mathrm{E}[\mathbf{x}]$. Note that the Absolute Risk Aversion for a CRRA utility function has the form $A_{\alpha}(W)=\frac{\alpha}{W}$. Hence as $W$ goes to infinity and $\alpha$ is bounded (no extreme risk aversion) $A_{\alpha}(W)$ tends to zero. This implies risk neutrality and hence $\lim _{W \rightarrow \infty} S(W, \mathbf{x})=\lim _{W \rightarrow \infty} B(W, \mathbf{x})=\mathrm{E}[\mathbf{x}]$ irrespective of the relative risk aversion coefficient.

\section{Proof of Lemma 1}

I prove first that $B^{\prime}(W)<1$. From the definition of buying price using implicit function formula: $\frac{d B}{d W}=1-\frac{U^{\prime}(W)}{\mathrm{E} U^{\prime}(W+\mathbf{x}-B(W, \mathbf{x}))}$ Since utility function is strictly increasing it must be that $\frac{d B}{d W}<1$.

Now I prove that $S^{\prime}(W-B(W))=\frac{B^{\prime}(W)}{1-B^{\prime}(W)}$ and $S^{\prime}(W-B(W))>B^{\prime}(W)$. From Lemma 6 (12), using the chain rule of differentiation, I have $B^{\prime}(W)=$ $S^{\prime}(W-B(W))\left(1-B^{\prime}(W)\right)$. Rearranging gives $S^{\prime}(W-B(W))=\frac{B^{\prime}(W)}{1-B^{\prime}(W)}$ Since $0<$ $B^{\prime}(W)<1$ by the above argument and Proposition 1.3 , I obtain $S^{\prime}(W-B(W))>$ $B^{\prime}(W)$.

Similarly I prove that $B^{\prime}(W+S(W))=\frac{S^{\prime}(W)}{1+S^{\prime}(W)}$ and $B^{\prime}(W+S(W))<S^{\prime}(W)$. Using Eq. 13 from Lemma 6, I have $S^{\prime}(W)=B^{\prime}(W+S(W))\left(1+S^{\prime}(W)\right)$ and hence $B^{\prime}(W+S(W))=\frac{S^{\prime}(W)}{1+S^{\prime}(W)}$ Since $S^{\prime}(W)>0$ by Proposition 1.3 , I get $B^{\prime}(W+$ $S(W))<S^{\prime}(W)$.

Now I will prove that $S^{\prime}(W)=\frac{S(W)-B(W)}{B(W)}$ for small positive $S(W)$. And by Proposition $1.3 S(W)>B(W)>0$. So when $S(W)$ is small and positive, then also $B(W)$ is small and positive. By Lemma $6(12), S(W-B(W))=B(W)$. For small $B(W)$ using first order Taylor expansion $B(W)=S(W)-\frac{d S}{d W} B(W)$ and hence it follows that $S^{\prime}(W)=\frac{S(W)-B(W)}{B(W)}$. Similarly, by Lemma $6(13), B(W+S(W))=$ $S(W)$. Hence, for small $S(W)$ using first order Taylor expansion $S(W)=B(W)+$ $\frac{d B}{d W} S(W)$ and it follows that $B^{\prime}(W)=\frac{S(W)-B(W)}{S(W)}$.

\section{Proof of Proposition 2.3}

Without loss of generality I assume that $\min (\mathbf{x})=0$. Fix $\mathbf{x}$ such that $\min (\mathbf{x})=0$. By Proposition 2.1, $B(W)$ and $S(W)$ are positive and hence by Proposition 1.3 $\tau(W)$ is positive over the whole range. Notice that range of $\tau(W)$ is determined by Proposition 2.1. If the domain of $S(W)$ is denoted $D_{S}$ and the domain of $B(W)$ 
is denoted $D_{B}$, then the domain of $\tau(W)$ is just $D_{S} \cap D_{B}=D_{B}$. In particular, for $\alpha \geq 1$ the domain of $\tau(W)$ is the interval $(0, \infty)$ and for $\alpha \in(0,1)$, the domain is the interval $\left(W_{L}(\mathbf{x}), \infty\right)$, where $W_{L}(\mathbf{x})$ is defined as in Proposition 2.1. To prove the proposition I have to check whether the following expression is negative:

$$
\tau^{\prime}(W)=\frac{S(W)}{B(W)}\left[\frac{S^{\prime}(W)}{S(W)}-\frac{B^{\prime}(W)}{B(W)}\right]
$$

From Lemma 6 I have the following equations: $B(W)=S(W-B(W))$, and $S(W)=B(W+S(W))$ For the proof, first order effects are not sufficient, but it turns out second order effects are. Therefore, by Taylor expansion of the second order I get from the above equations: $B(W)=S(W)-S^{\prime}(W) B(W)+S^{\prime \prime}(W) B^{2}(W)$ and $S(W)=B(W)+B^{\prime}(W) S(W)+B^{\prime \prime}(W) S^{2}(W)$. I only need to check the difference from Eq. 15 which I can rewrite as follows using the above Taylor expansions:

$$
\begin{aligned}
\frac{S^{\prime}(W)}{S(W)}-\frac{B^{\prime}(W)}{B(W)} & =\frac{\frac{S(W)-B(W)}{B(W)}+S^{\prime \prime}(W) B(W)}{S(W)}-\frac{\frac{S(W)-B(W)}{S(W)}-B^{\prime \prime}(W) S(W)}{B(W)} \\
& =\frac{S^{\prime \prime}(W) B^{2}(W)+B^{\prime \prime}(W) S^{2}(W)}{S(W) B(W)}<0
\end{aligned}
$$

where the last inequality follows from the fact that both $B(W)$ and $S(W)$ are concave (by Lemma 2) and nonnegative (by Proposition 2.1).

\section{Proof of Proposition 3.1}

Take any non-degenerate lottery $\mathbf{y}$ with $S(W, \mathbf{y})>B(W, \mathbf{y})$. Such a lottery exists by Proposition 1.3. I can find a sequence of real numbers which all are greater than $B(W, \mathbf{y})$ and smaller than $S(W, \mathbf{y})$. I can then treat these numbers as a support for a new lottery $\mathbf{x}$. I assign probabilities to each of these numbers such that they sum to one and are positive for at least two of these numbers (such that the resulting lottery is non-degenerate). Suppose I choose $n$ such numbers. By Proposition 1.1 I can now conclude that $S(W, \mathbf{y})>\max _{i \in\{1, \ldots, n\}} x_{i}>\mathrm{E}[\mathbf{x}]>S(W, \mathbf{x})>B(W, \mathbf{x})>$ $\min _{i \in\{1, \ldots, n\}} x_{i}>B(W, \mathbf{y})$.

\section{Proof of Proposition 3.2}

Suppose that at any wealth $W \in[\underline{w}, \bar{w}]$ the decision maker prefers lottery $\mathbf{x}$ to lottery $\mathbf{y}$ in a direct choice but assigns higher certainty equivalent to lottery $\mathbf{y}$. Given such a pattern of preferences it is easy to design an arbitrage strategy that extracts at least $W-\underline{w}$ from this decision maker. Suppose $W \in[\underline{w}, \bar{w}]$ is an initial wealth. Construct a sequence $W_{i}, i \in\{1,2, \ldots, n\}$ such that:

- $W_{0}=W$

- $W_{i}=W_{0}-\sum_{k=1}^{i} \epsilon_{k}, \epsilon_{i}>0 i \in 1,2, \ldots, n$

- $W_{n} \geq \underline{w}, \quad W_{n+1}<\underline{w}$

- for $i$ even (including 0) $W_{i+1}+\mathbf{x} \succ W_{i}+\mathbf{y}$

- for $i$ odd: $C E\left(W_{i}+\mathbf{x}\right)<C E\left(W_{i+1}+\mathbf{y}\right)$ 
Notice that such a sequence exists by monotonicity and continuity of preferences and by properties of real numbers. Assume w.l.o.g. that $W_{0}+\mathbf{y} \succ W_{0}$. The arbitrage strategy is now the following:

0) Take $\mathbf{y}$

1) Exchange $\mathbf{y}$ for $\mathbf{x}$ and pay me $\epsilon_{1}$

2) Exchange $\mathbf{x}$ for $C E\left(W_{1}+\mathbf{x}\right)-W_{1}$

3) Exchange $C E\left(W_{1}+\mathbf{x}\right)$ for $C E\left(W_{1}+\mathbf{y}\right)$ and pay me $\epsilon_{2}$

4) Exchange $C E\left(W_{2}+\mathbf{y}\right)-W_{2}$ for $\mathbf{y}$

5) Repeat

The above arbitrage strategy extracts the amount of wealth equal to $W-\underline{w}$ from the decision maker.

\section{Proof of Proposition 3.3}

In what follows I will try to construct an arbitrage strategy to exploit the decision maker and show that it is not possible. Given a DARA utility function $U$, take $\mathbf{x}$ such that $B(W, \mathbf{x})<0$. I will examine only this case since in the other cases the proof is trivial.

Suppose first, the decision maker initially has non-random position $W$. If the price $b$ for the lottery is bigger than $B(W, \mathbf{x})$, the decision maker will not buy it. Hence, a price which is a part of an arbitrage strategy must be smaller than $B(W, \mathbf{x})$. Given such price $b$, the decision maker buys the lottery. His new position is $W+\mathbf{x}-b$. If the price $s$ is smaller than $S(W-b, \mathbf{x})$, then the decision maker does not want to sell. Hence a price which is a part of an arbitrage strategy must be bigger than $S(W-b, \mathbf{x})$. By Proposition 1.3, I know that $S$ is strictly increasing and $b<B(W, \mathbf{x})$. Therefore:

$$
s>S(W-b, \mathbf{x})>S(W-B(W, \mathbf{x}), \mathbf{x})=B(W, \mathbf{x})>b
$$

where the equality follows from Lemma 6 (12).

Suppose now, that the decision maker initially has a random position $W+\mathbf{x}$. By the same argument as above the price $s$, which is a part of an arbitrage strategy has to be greater than $S(W, \mathbf{x})$, otherwise the decision maker would not sell the lottery x. After selling the lottery, the decision maker's new position is $W+s$. The price $b$ which is a part of an arbitrage strategy has to be smaller than $B(W+s, \mathbf{x})$. By Lemma 1 , I know that $\frac{\partial B(W, \mathbf{x})}{\partial W} \leq 1$ for all $W \geq 0$. Hence:

$$
s-S(W, \mathbf{x})>B(W+s, \mathbf{x})-B(W+S(W, \mathbf{x}), \mathbf{x})
$$

By Lemma 6 (13), I know that $B(W+S(W, \mathbf{x}), \mathbf{x})=S(W, \mathbf{x})$, and hence:

$$
s>B(W+s, \mathbf{x})>b
$$

That proves that with decision maker's initial position equal to either $W$ or $W+\mathbf{x}$, all arbitrage strategies have the property that $s>b$. However, this cannot be an arbitrage strategy since it makes negative profit equal to $b-s$. This proves that there are no arbitrage strategies. 


\section{References}

Bell, D.E. (1982). Regret in decision making under uncertainty. Operations Research, 30, 961-981.

Chilton, S., Jones-Lee, M., McDonald, R., Metcalf, H. (2012). Does the WTA/WTP ratio diminish as the severity of a health complaint is reduced? Testing the smoothness of the underlying utility of wealth function. Journal of Risk and Uncertainty, 45, 1-24.

Cox, J.C., \& Sadiraj, V. (2006). Small- and large-stakes risk aversion: implications of concavity calibration for decision theory. Games and Economic Behavior, 56, 45-60.

Foster, D., \& Hart, S. (2009). An operational measure of riskiness. Journal of Political Economy, 117, 758-814.

Fudenberg, D., \& Levine, D.K. (2006). A dual-self model of impulse control. American Economic Review, 96, 1449-1476.

Grether, D.M., \& Plott, C.R. (1979). Economic theory of choice and the preference reversal phenomenon. American Economic Review, 69, 623-638.

Gul, F. (1991). A theory of disappointment aversion. Econometrica: Journal of the Econometric Society, $59,667-686$.

Hanemann, W.M. (1991). Willingness to pay and willingness to accept: how much can they differ? American Economic Review, 81, 635-647.

Horowitz, J.K., \& McConnell, K.E. (2002). A review of WTA/WTP studies. Journal of Environmental Economics and Management, 44, 426-447.

Kahneman, D., \& Tversky, A. (1979). Prospect theory: an analysis of decision under risk. Econometrica, 47, 263-292.

Knetsch, J.L., \& Sinden, J.A. (1984). Willingness to pay and compensation demanded: experimental evidence of an unexpected disparity in measures of value. The Quarterly Journal of Economics, 99, 507521.

Lewandowski, M. (2011). Risk attitudes, buying and selling price for a lottery and simple strategies. http:// ssrn.. com/abstract $=2189406$.

Loomes, G., \& Sugden, R. (1982). Regret theory: an alternative theory of rational choice under uncertainty. Economic Journal, 92, 805-824.

Palacios-Huerta, I., \& Serrano, R. (2006). Rejecting small gambles under expected utility. Economics Letters, 91, 250-259.

Rabin, M. (2000). Risk aversion and expected-utility theory: a calibration theorem. Econometrica, 68(5), $1281-1292$.

Raiffa, H. (1968). Decision analysis: introductory lectures on choices under uncertainty. Addison-Wesley.

Rubinstein, A. (2006). Dilemmas of an economic theorist. Econometrica, 74, 865-883.

Safra, Z., \& Segal, U. (2008). Calibration results for non-expected utility theories. Econometrica, 76, $1143-1166$.

Schmidt, U., Starmer, C., Sugden, R. (2008). Third-generation prospect theory. Journal of Risk and Uncertainty, 36, 203-223.

Thaler, R. (1980). Toward a positive theory of consumer choice. Journal of Economic Behavior and Organization, 1(1), 39-60.

Viscusi, W.K., \& Huber, J. (2012). Reference-dependent valuations of risk: why willingness-to-accept exceeds willingness-to-pay. Journal of Risk and Uncertainty, 44, 19-44.

von Neumann, J., \& Morgenstern, O. (1944). Theory of games and economic behavior. Princeton: Princeton University Press.

Yaari, M.E. (1987). The dual theory of choice under risk. Econometrica, 55(1), 95-115. 